\title{
Le dire et le faire - Le registre du don dans la communication des entreprises
}

Dominique Kohler

\section{(2) OpenEdition}

12 Journals

Édition électronique

URL : http://journals.openedition.org/communicationorganisation/2588

DOI : 10.4000/communicationorganisation.2588

ISSN : $1775-3546$

Éditeur

Presses universitaires de Bordeaux

Édition imprimée

Date de publication : 1 novembre 2001

ISSN : 1168-5549

Référence électronique

Dominique Kohler, "Le dire et le faire - Le registre du don dans la communication des entreprises ", Communication et organisation [En ligne], 20 | 2001, mis en ligne le 27 mars 2012, consulté le 04 mai 2019. URL : http://journals.openedition.org/communicationorganisation/2588 ; DOI : 10.4000/ communicationorganisation. 2588

Ce document a été généré automatiquement le 4 mai 2019.

(c) Presses universitaires de Bordeaux 


\title{
Le dire et le faire - Le registre du don dans la communication des entreprises
}

\author{
Dominique Kohler
}

1 Les entreprises doivent constamment faire face à un double défi : être un carrefour exemplaire de la vie sociale et participer à la vie de la société aux côtés de l'État providence. Partenaires des pouvoirs publics, des associations, des médias, il leur est demandé de relancer l'économie, le commerce extérieur, de créer des emplois mais aussi d'être un lieu de formation et d'épanouissement individuel ; il leur est demandé en plus de respecter l'environnement non seulement écologique mais culturel et social. Les entreprises ploient sous le fardeau des engagements les plus divers et il est vrai qu'elles assument de réelles responsabilités sociales. Les plus impliquées d'entre elles - telles les banques ou les assurances, les multinationales à haut risque de pollution, par exemple sont celles qui peuvent le moins se permettre d'éviter les problèmes de solidarité, d'environnement ou de santé : en interne, l'homme derrière le salarié questionne; en externe, la société derrière le client juge... L'analyse des comportements des uns et des discours des autres nourrit chaque jour une littérature abondante à propos de laquelle on est en droit de s'interroger. N'y a-t-il pas un paradoxe, voire une contradiction entre la logique économique de l'entreprise et sa recherche déclarée de valeurs sociales, culturelles et éthiques?

2 À en croire les acteurs et les observateurs de la vie économique, on peut envisager plusieurs explications : besoin de déculpabilisation, nouveaux marchés, résurgence d'un système, tournant social, fait de société, phénomène de mode... Mais devant les formes de solidarité actuelles, puissamment soutenues par les médias, peut-on dire que l'entreprise ne suit qu'un simple phénomène de mode: nouvelles valeurs ou nouveaux marchés de l'altruisme? Est-il question, plus profondément, d'une évolution des mentalités, de la réaction d'une société d'hyper-consommation où les entreprises entendent se déculpabiliser en endossant une responsabilité civile ? Et dans ce cas, peut-on penser que la recherche du profit est moins «coupable » à partir du moment où l'entreprise a découvert la façon de justifier ses moyens par des fins altruistes. On peut aussi émettre 
l'hypothèse de la résurgence d'un système économique et social, fondé sur les théories du don, au sujet duquel de nombreux chercheurs économistes et ethno-sociologues se sont interrogés par le passé : l'entreprise vérifiant qu'au-delà du seul profit matériel fondé sur la rentabilité, il existe une autre sorte de profit moral dont les retombées en termes d'image, de reconnaissance, d'intégration, de légitimité, sont tout aussi déterminantes pour son avenir; notre société serait-elle alors, à l'aube du XXIe siècle, au cœur d'un tournant social décisif, dont l'entreprise aurait su saisir le sens profond?

3 Ces nouvelles aspirations que recouvrent le social et le culturel conditionnent la politique des dirigeants d'entreprise et, c'est précisément en fonction de ces nouveaux besoins qu'ils ont fait évoluer leurs pratiques de communication tant à l'intérieur qu'à l'extérieur. Les activités de mécénat et de partenariat sont significatives des différents niveaux d'implication et de motivation des entreprises. Pourtant, il ne suffit pas d'appliquer des techniques de marketing et de traiter le mécénat ou tout autre forme de partenariat comme un outil de plus à leur service car, à la différence des autres techniques, toutes les retombées ne sont pas prévisibles, tous les résultats ne sont pas mesurables: autant d'inconnues qui font des pratiques généreuses une communication à risques... Ces observations conduisent à souligner la complexité des problèmes que pose le réenracinement des entreprises dans la société, et dans son tissu humain. Avec pour effet de réintroduire dans la démarche mercantile des éléments, des façons de faire, des discours de type affectif qui en accentuent l'ambiguïté, ces pratiques en viennent à se rapprocher de celles que mettaient en œuvre les sociétés dites primitives ou archaïques dans le cadre d'une économie du don : mais peut-on donner quand on vend... ?

Le mécénat recouvre différentes réalités : il est perçu par certains comme de la "publicité déguisée ", surtout quand l'opération est liée à un acte commercial : c'est l'administration des Postes et Télécommunications lorsqu'elle vend au bénéfice de la Croix Rouge des timbres à supplément. Pour d'autres, le mécénat correspond à des opérations de financement dont les retombées ne sont pas totalement philanthropiques, telle l'acquisition des « Tournesols » de Vincent Van Gogh par la société japonaise d'assurances Yasuda, mais l'absence de rendements réguliers pour de tels placements n'empêche pas les plus-values, notamment en terme d'image. Par contre, le mécénat peut impliquer l'entreprise sous une forme beaucoup plus directe que par de simples mouvements de fonds, qu'il s'agisse de partenariat en termes de coproduction de films, de mécénat croisé (le culturel au service de l'humanitaire) ou encore de mécénat de décentralisation et de proximité II existe bien des niveaux d'implication, par exemple: la Fondation pour le Mécénat Humanitaire regroupant, autour d'Axa, une trentaine d'entreprises qui ont pour volonté déclarée de mobiliser leur personnel à travers un projet fédérateur et qui souhaitent se positionner comme "entreprise citoyenne, responsable et solidaire". L'objectif consiste à "développer un véritable partenariat entre entreprises et organismes humanitaires", la Fondation jouant le rôle de "conseil et d'organe d'ingénierie humaine de ses membres». Le montant moyen des opérations de mécénat conduites à travers la Fondation depuis sa création en 1986 dépasse 8 MF chaque année... mais avec quels résultats?

5 Les retombées du mécénat - qu'il soit outil de communication, facteur de cohésion interne, ciment d'une responsabilité sociale,... - font l'objet de différentes mesures, mais aux dires des intéressés eux-mêmes, rien de très performant n'existe qui permette de mesurer l'effet réel des actions conduites: les initiatives de mécénat humanitaire modifient-elles réellement la vie sociale, le comportement des partenaires publics et 
associatifs, l'attitude des consommateurs? Un de nos objectifs a été précisément d'évaluer ${ }^{1}$ les incidences des choix des entreprises et l'influence réciproque des relations instaurées avec les associations, les institutions, les pouvoirs publics, les consommateurs. Comment expliquer que les discours mais aussi les actions des entrepreneurs ne semblent pas toujours bien compris et partagés: "c'est un problème de flou: on ne sait pas vraiment quelles sont les retombées de toutes ces actions... Il n'existe pas d'outil de mesure et lorsqu'on décortique l'image de l'entreprise, il est impossible de savoir précisément ce qui provient des campagnes de publicité produits, de la communication institutionnelle, du charisme du dirigeant, du comportement des salariés à l'interne et à l'externe, a fortiori donc du mécénat ». Cette remarque du Secrétaire général de l'Admical résume assez bien une opinion largement partagée par ceux qui sont concernés par cette nouvelle " citoyenneté » de l'entreprise. Beaucoup trop d'opérations se soldent par des échecs parce que les responsables ne reçoivent pas de réponses appropriées aux questions fondamentales qu'ils se posent. Autant les motivations des entreprises sont considérées comme facilement identifiables et sujettes à des analyses précises, autant les retombées des actions généreuses du mécénat sont réputées difficiles à mesurer ou même insaisissables. Comment dès lors établir avec certitude une relation de cause à effet entre actions et résultats? Comment évaluer en complément des autres moyens d'action et en termes de bénéfices acquis rapportés aux budgets engagés et aux objectifs fixés, l'influence de la variable mécénat sur les résultats globaux de l'entreprise? Comment mesurer les impacts de notoriété et d'image? Les entreprises ont abordé le problème en essayant de quantifier les retombées dans la presse, en organisant des enquêtes en leur propre sein ou en procédant à des sondages auprès du public: le degré de notoriété est mesuré au moyen de pré-tests et de post-tests permettant de déceler des écarts de notoriété mais ces mesures se heurtent ici encore aux mêmes difficultés que précédemment : comment isoler la variable mécénat des autres actions promotionnelles qui ont eu lieu en même temps?

6 La Fondation Gan, par exemple, avait effectué à l'occasion de la «Fête du Cinéma » un sondage à Paris et en province, auprès d'un échantillon représentatif du public qui fréquente, occasionnellement ou régulièrement, les salles. L'enquête entendait mesurer : «la notoriété de la Fondation Gan, en soi et comme sponsor de la «Fête », le contenu prêté à ses actions et l'agrément à ce contenu, l'attribution de la fête à la Fondation ou au Gan et les effets de cette attribution sur l'image de l'une et l'autre ». Le rapport concluait "quels que soient les items considérés, les résultats obtenus sont homogènes " et précisait : « c'est parce que la Fondation Gan apparait généreuse et aimant le cinéma [...] qu'on adhère à son action et qu'on en est reconnaissant au Gan »; les conclusions de l'enquête restaient très générales et le commentaire final se contentait d'admettre une relation de cause à effet entre générosité et reconnaissance, ce qui ressemble a priori à une lapalissade... Cependant, comme nous aurons l'occasion de l'approfondir par la suite en traitant de l'univers du don, l'entreprise se trouve engagée avec le mécénat dans des relations d'interdépendance propres à cet univers et il n'est pas si sûr que la générosité entraîne toujours de la reconnaissance... L'univers du don se comporte comme "un système, un tout organisé » où chacun des éléments en interaction engendre des relations extrêmement complexes qui ne peuvent pas se résumer au schéma simplifié appliqué au Gan : « donner - recevoir - être reconnaissant ».

7 Quant à connaître les conséquences en interne sur le climat de l'entreprise, en termes d'adhésion ou de participation du personnel, voire des syndicats, cela paraît tout aussi 
difficile... Tous les efforts entrepris pour informer le personnel puis pour l'impliquer dans un projet social, culturel ou humanitaire risquent souvent d'être anéantis par l'absence de publication de résultats. Les valeurs culturelles fortes suscitent des attentes de même intensité : d'où la nécessité de vérifier que les actions concrètes de l'entreprise sont bien à la mesure de ces attentes. Différents indicateurs permettent d'établir un bilan interne afin de mesurer le degré de satisfaction des membres du personnel : il peut s'agir de la baisse du taux d'absentéisme par exemple, de la fidélité du personnel qualifié, de la paix sociale dans l'entreprise, de l'évolution du bien-être dans et hors de l'entreprise, mais aussi du nombre de candidatures à l'embauche ou de la qualité du recrutement... Mais l'imprécision des mesures des retombées, l'équivoque des pratiques, l'ambiguïté des motivations font du mécénat un moyen difficile à maitriser voire même, dans certains cas, non exempt de risques.

Certains types d'actions courent le risque d'être perçus par une partie du personnel comme une contrainte, résultat d'une politique paternaliste - «donner aux salariés du pain et des jeux » - où le salarié se sent pris au piège par un patron à qui l'on doit tout, risque que l'on peut appeler de la "double dépendance »: l'adhésion à des actions de mécénat s'ajoutant aux obligations du contrat de travail que le salarié a déjà contractées. S'il s'agit d'actions sociales, culturelles ou humanitaires, le succès dépendra des motivations mêmes de l'entreprise avec lesquelles le projet doit rester cohérent, c'est-àdire sous réserve que la valeur de ses actions cadre bien avec ses activités, avec son image externe et avec les aspirations de ses salariés. Quant aux risques externes, ils ne sont pas moins complexes. L'incidence d'une actualité imprévue, d'une mauvaise présentation journalistique ou d'un courant d'opinion fortuit, provoqué ou non, peut détériorer pour longtemps une image, avec une remarque du type « on ne construit pas son image sur le malheur des autres ». Un projet peut aussi manquer son but en raison de la notoriété d'un interprète, ce que les publicitaires appellent un effet de "vampirisation» ou de « cannibalisme».

9 Pourtant l'entreprise se doit de relever de nouveaux défis qui, sous la pression conjuguée de l'opinion publique, des consommateurs, des associations et des institutions gouvernementales, l'engagent à concilier croissance économique, utilité commerciale et intérêt général. Le désengagement de l'État, la crise de légitimité des institutions, la perte des valeurs traditionnelles liés à un pragmatisme général, la complexification du tissu social et économique, la concurrence du marché européen, le poids de l'opinion publique... sont autant d'incidences qui contribuent à conforter l'entreprise dans son nouveau rôle de citoyenne. Mais quelles sont alors les limites de l'engagement? Jusqu'où aller pour ne pas être soupçonné de manipulation? Les impératifs économiques ont longtemps enfermé les entreprises dans une logique du profit alors que l'essor des théories managériales a conduit les entrepreneurs à prendre conscience de l'Autre dans un contexte socioculturel plus soucieux de l'humain. Cette nouvelle responsabilité sociale constitue en réalité pour l'entreprise une valeur ajoutée. L'intérêt général est devenu une composante du professionnalisme de l'entreprise et un critère de qualité qui vient s'ajouter à ceux de rentabilité, de sécurité, de performance, de progrès... Mais en décidant d'axer sa politique institutionnelle sur le mécénat de solidarité plutôt que sur le sponsoring sportif, en optant pour le partenariat, l'entreprise engage sa communication dans un processus complexe mal connu, étudié souvent de façon trop dogmatique, qui appartient à l'univers du don. 
10 Il est vrai que le terme « communication » est polysémique et protéiforme. Son contenu sémantique a beaucoup évolué au cours de son histoire mais moins que les domaines auxquels il a été appliqué. Les cybernéticiens, biologistes, anthropologues, linguistes, sociologues ou psychiatres - qu'ils s'appellent Norbert Wiener, L. Von Bertalanfy, Edward T. Hall, Paul Watzlawick, Gregory Bateson - ont tous contribué à faire en sorte que ce mot fourre-tout rassemble aujourd'hui des moyens, des compétences, des techniques et des théories. Son incursion dans l'entreprise a généré des spécialistes, un département, un processus social permanent intégrant de multiples modes de comportement qui concernent les partenaires, les consommateurs, la société. Ce que demandent en fait les entrepreneurs concernés, sans le formuler explicitement, c'est de comprendre finalement les mécanismes de l'engagement qu'enclenche une politique de communication de solidarité. C'est bien le problème qui se cache derrière leur souci de ne pas maîtriser tous les paramètres décisionnels en matière de mécénat, d'engager un acte de communication dans un processus dont ils ne contrôlent pas tous les effets, de pressentir que les frontières du domaine de la générosité - c'est-à-dire de l'univers $d u$ don - sont aussi floues que les concepts qui le structurent. Définir ces concepts est donc le point de passage obligé de toute analyse cherchant à comprendre sur quel registre joue l'entreprise dite citoyenne.

11 Le thème de l'écologie, né au cœur de la contestation générale des années soixante-dix, n'est pas nouveau en soi : la société de consommation a depuis longtemps récupéré l'idéologie du «naturel» au profit de nombreux secteurs commerciaux. S'agissant non plus seulement de «naturel» mais de la nature altérée (l'alimentation, les matières premières, l'énergie, le climat...), l'environnement écologique, mais aussi culturel et social, se rappelle à l'homme qui en porte plus que jamais la responsabilité. Facteur de synergie sociale, l'environnement au sens large du terme est désormais un problème global qui implique la mobilisation de tous les acteurs de la société. Mais lorsqu'il s'agit des entreprises, comment concilier l'économie et l'écologie que tout sépare? Avec l'écologie pour cas d'étude, il s'est agi d'analyser en quels termes les entrepreneurs et les hommes de communication ont justifié leur positionnement; positionnement quelque peu inconfortable au sommet de cette vague de sens civique qui déferle depuis plusieurs années sur notre société et entraîne avec elle l'entreprise alors que celle-ci hier encore naviguait, pour reprendre l'image de Karl Marx, "sur les eaux glacées du calcul égoïste »... Les entrepreneurs ont été les premiers à conduire des études portant sur leur politique face aux contraintes écologiques afin de montrer que leur volonté de concilier le développement économique et le développement durable était bien réelle : «plus de la moitié des industriels pensent que la prise en compte de l'écologie induit des effets bénéfiques sur le plan technique, qu'elle est facteur de progrès social» (Le Monde), l'écologie "représente un argument commercial puissant », des avantages économiques et des retombées en terme d'image institutionnelle. Bernard Tramier, Directeur Environnement de Elf, écrivait déjà en 1990 : «cela fait vingt ans au moins que les industriels dans leur ensemble, et Elf Aquitaine en particulier, ont beaucoup investi en faveur de l'environnement, afin d'en préserver la qualité tout en remplissant leur rôle économique ». Il précisait que son groupe consacrait «à la sécurité des hommes et à la protection de l'environnement»1,5 milliards de francs depuis 1988 et il citait l'amélioration des rejets de matières oxydables au plan national qui ont baissé de $70 \%$ en dix ans... 


\begin{tabular}{|c|c|c|c|c|c|}
\hline Registre & Objectifs & Actions & $\begin{array}{c}\text { Valeurs } \\
\text { véhiculées }\end{array}$ & Attentes & $\begin{array}{c}\text { Valorisa- } \\
\text { tion et } \\
\text { Attitude }\end{array}$ \\
\hline $\begin{array}{l}\text { Econo- } \\
\text { mique }\end{array}$ & $\begin{array}{l}\text { Confirmer sa } \\
\text { vocation } \\
\text { Etablir une } \\
\text { cohérence avec le } \\
\text { métier } \\
\text { Convaincre, } \\
\text { Justifier, } \\
\text { Expliquer }\end{array}$ & $\begin{array}{l}\text { Investissement } \\
\text { réfléchi }\end{array}$ & \begin{tabular}{|l} 
Qualité \\
Compétence \\
Performance \\
Cohérence
\end{tabular} & $\begin{array}{l}\text { Retour sur } \\
\text { investissement } \\
\text { Identité } \\
\text { Notoriété }\end{array}$ & $\begin{array}{l}\text { Charisme } \\
\text { Se justifier }\end{array}$ \\
\hline $\begin{array}{l}\text { Moral } \\
\text { civique }\end{array}$ & \begin{tabular}{|l|} 
Affirmer son \\
identité \\
Améliorer son \\
image \\
Expliquer
\end{tabular} & $\begin{array}{l}\text { Partenariat, } \\
\text { Mécénat, } \\
\text { Association, } \\
\text { Conseil, Partage } \\
\text { des moyens et des } \\
\text { savoir-faire de } \\
\text { l'entreprise, } \\
\text { Coopération, } \\
\text { Echange }\end{array}$ & $\begin{array}{l}\text { Responsabilité } \\
\text { Aide, } \\
\text { Compréhension } \\
\text { Conciliation } \\
\text { Notion de service } \\
\text { rendu }\end{array}$ & $\begin{array}{l}\text { Reconnaissance } \\
\text { Capital de } \\
\text { sympathie } \\
\text { Audience }\end{array}$ & $\begin{array}{l}\text { Solidarité } \\
\text { Se } \\
\text { valoriser }\end{array}$ \\
\hline Social & $\begin{array}{l}\text { Mobiliser le } \\
\text { personnel } \\
\text { Impliquer tous les } \\
\text { acteurs de } \\
\text { l'entreprise } \\
\text { Responsabiliser } \\
\text { Eduquer } \\
\text { Rassurer }\end{array}$ & $\begin{array}{l}\text { Information } \\
\text { Formation } \\
\text { Communication } \\
\text { Ecoute et } \\
\text { dialogue, } \\
\text { Participation }\end{array}$ & $\begin{array}{l}\text { Engagement } \\
\text { Respect } \\
\text { Ouverture } \\
\text { Transparence }\end{array}$ & $\begin{array}{l}\text { Ecoute } \\
\text { Harmonie } \\
\text { Climat } \\
\text { Image }\end{array}$ & $\begin{array}{l}\text { Valeurs } \\
\text { spirituelles } \\
\\
\text { S'associer }\end{array}$ \\
\hline
\end{tabular}
publicitaire parue en 1991 - est construit sur le même registre : "comme nous avons commencé cette politique au début de la décennie précédente, une comparaison avec l'année 1981 s'impose : diminution du degré de pollution des eaux résiduaires de plus de $50 \%$ en 10 ans, baisse des métaux lourds entre 62 et $96 \%$, réduction de $67 \%$ des matières organiques contenues dans les eaux résiduaires». D'où ce slogan: «Bayer. Etre compétent c'est aussi être responsable ». À l'envi, dans leurs campagnes institutionnelles, Mazda se déclare «Sur le chemin de la civilisation » et Toshiba s'affirme « Partenaire de l'aventure humaine "... Quarante grandes entreprises avaient accepté l'invitation du ministère de l'environnement à élaborer « un plan environnement » comprenant non seulement un engagement écrit mais aussi un "écobilan », dont les retombées sont des réalisations très concrètes : remplacement des chlorofluorocarbones, essence sans plomb, investissement dans les technologies propres, recyclage ou bio-dégradabilité des produits d'emballage, élimination du phosphate dans les lessives, etc. Les entreprises cherchent ainsi à rejeter la culpabilité dont on les accable généralement. Autrement dit: Responsables, oui, mais pas coupables... Elles entendent se laver des accusations coutumières portées contre elles sur un triple front - celui de l'argent et du profit, de l'égoïsme et de la pollution - dressé au nom de la protection de l'espèce et de son environnement contre une technologie ennemie de l'humain. Le positionnement des entreprises, délibérément tourné vers l'Autre est valorisant.

Le geste de donner a sans doute été la première, la plus archaïque, la plus essentielle de toutes les formes de communication: je donne, donc je dis qui je suis. Cependant, les stratégies des entreprises qui consistent à s'approprier un rôle de bienfaiteur, à communiquer sur le thème de la solidarité suscitent des réticences quant aux motivations, voire un certain scepticisme quant aux résultats. L'équivoque des pratiques et des discours pourrait provenir en partie de l'acte de donner lui-même qui est étymologiquement et sociologiquement porteur d'ambiguïté ? Entendons par là le fait que 
toutes les actions de mécénat ou de solidarité, toutes les formes de don matérielles ou non appartiennent certes à un même univers mais ne sont pas liées à un même registre motivationnel. En effet, si les motivations restent complexes et souvent enchevêtrées, les discours des entrepreneurs se regroupent en trois grands registres majeurs, suivant que l'entreprise cherche à se justifier, se valoriser ou s'humaniser: le registre d'ordre économique valorise les activités de l'entreprise, met l'accent sur sa bonne santé financière, donc sa pérennité, rassure aussi bien les actionnaires que le personnel; le registre d'ordre moral, civique prétend faire respecter une éthique qui garantit la transparence et la sauvegarde de l'intérêt général, dans la recherche d'une vie plus saine et plus féconde ; quant au registre d'ordre social, il défend le bien-fondé des activités de l'entreprise vis-à-vis de son personnel et de ses publics, tout en garantissant le dialogue, le respect des autres, le partage du savoir et des acquis nouveaux. Suivant que l'entreprise joue de l'un ou de l'autre de ces registres et bien souvent de plusieurs à la fois, on comprend l'ambiguïté des motivations relevée et la difficulté d'y retrouver un fondement altruiste. Sorte de clignotant social, l'entreprise est-elle ce lieu de rémanence de la mythologie du don, mode de communication spécifique, construit sur une ambiguïté, fonction elle-même des motivations et des implications des acteurs en cause ? L'acte de donner implique des discours et des pratiques fortement connotés instituant des relations complexes. Le caractère oblatif rejaillit sur des actions de même nom mais pas de même nature...

14 L'observation et l'étude de la circulation des biens dans les sociétés primitives ont convergé vers une analyse du don en tant que "phénomène total ", c'est-à-dire à la fois religieux, économique, politique, juridique et familial. Bien sûr, les caractéristiques des échanges ont évolué avec le perfectionnement de l'organisation des groupes sociaux et il est d'obligation de faire référence aux travaux des anthropologues, ethnologues, sociologues et économistes qui en ont traité. S'il est intéressant de prendre en compte les expériences de Marcel Mauss, de Franz Boas, de Bronislaw Kaspar Malinowski ainsi que les analyses de Claude Levi-Strauss ou de Jean Cazeneuve, c'est qu'une telle approche permet de dégager des éléments permanents de l'univers du don, tels que «la valeur sociale ", « la force intrinsèque des choses » ou le "potlatch »... La connaissance de ces théories, fondées sur des données anthropologiques, facilite l'analyse de certaines règles qui régissent toujours notre société ; appliquées au domaine de l'entreprise, ces règles contribuent à faire du don un mode de communication particulier. Par exemple, la Sollac a adopté pour son éthique de société des valeurs de base au nombre de six, caractéristiques des codes et domaines motivationnels chers aux ethnologues :

15 - le « respect des personnes et de leur différence, reconnaissance de chacun dans son engagement » et la "vérité car la connaissance des faits détermine notre performance et notre responsabilité », soit le Code de l'honneur ;

16 - l'«équité car les contributions individuelles et collectives doivent trouver une contrepartie comme la rémunération et la considération ", c'est-à-dire le Principe de réciprocité;

17 - l'«écoute qui est marque de considération, de respect et source de proposition, de progrès et de convivialité » et l'« effort et innovation pour apporter à chaque instant une contribution supplémentaire au projet de l'entreprise et assurer ainsi sa pérennité et son développement ", soit la Sauvegarde des liens sociaux; 

(horizontalement) le double aspect de la nature du don : le service de type charismatique, la proposition de registre trivial. C'est la combinaison de ces axes qui détermine le registre des relations entre donateur et donataire : pour l'individu, le don est ce qu'il possède à titre personnel inaliénable et qui peut être soit inné (y compris son patrimoine génétique), soit acquis en fonction de ses qualités de réceptivité, c'est-à-dire de savoir-faire. Quant aux rapports avec les autres, il convient de distinguer entre l'objectif et le subjectif, et parler soit de proposition (ou d'offre) touchant plus directement le réel (sans pour autant être privée d'une composante imaginaire mais qui est ici secondarisée), soit de service rendu à l'autre (un service qui peut porter sur du concret) dont la dominante est d'ordre relationnelle et communicationnelle. L'amalgame, voulu ou non, de ces principales caractéristiques ne peut être que source d'incertitudes et d'ambiguïté, en particulier dans le cas du don pratiqué vis-à-vis des autres, suivant que l'on vise la dimension stratégique de l'offre ou le service à rendre, et que l'on y inclut une motivation sous-jacente qui est celle de lui faire jouer le rôle de vecteur de communication (plan subjectif). La notion de communication est en ce sens fondamental. Gratuit ou non, le don permet de maintenir entre les hommes des liens, qui ne sont pas uniquement d'ordre économique.

21 L'action de donner suppose normalement l'acte de recevoir, c'est-à-dire accepter, prendre ce qui est offert, l'accueillir, le retenir, l'admettre ou l'agréer, autant de termes qui montrent que l'opération n'est pas passive. 


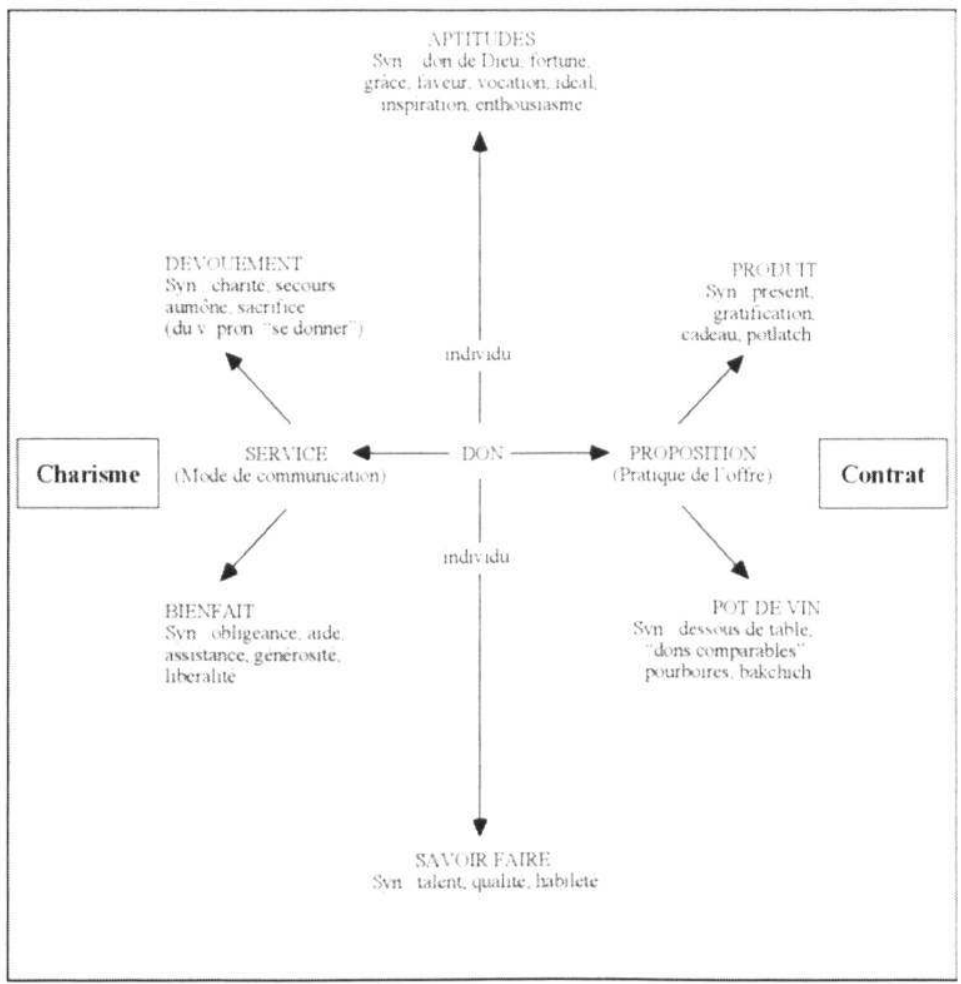

Schéma fonctionnel de l'univers du don

De même que l'action de donner fluctue entre l'idée de sacrifice et celle de transaction, l'action de recevoir participe d'une même ambivalence entre l'accueil et l'accord: l'obligation de recevoir crée une sorte de lien interne et magique entre les objets et les hommes et confère au don une valeur sociale. Le don, pensé et vécu comme un acte de communication stratégique, enclenche une dynamique irréversible dont les conséquences sont importantes pour toutes les relations, tant internes qu'externes, de l'entreprise. La relation donatrice met en jeu deux courants de force symétriques : l'un centrifuge, qui porte vers les autres, à travers des liens à la fois triviaux (socio-éco-culturels) et transcendants (idéologie, religion, partage), l'autre centripète qui ramène vers le donateur à titre de contre-don, tous les avantages que mérite son action. L'aspect altruiste de la communication s'équilibre avec l'aspect égocentrique. Cet échange dynamique complexe, qui, à l'origine, était l'apanage des sociétés primitives, devient ainsi l'élément d'une stratégie de communication, d'où l'ambiguïté permanente et immanente du concept de don en entreprise, et cette représentation dynamique d'un circuit fermé entre l'individu et les autres, entre l'entreprise-personne et son environnement social ; circuit générateur de liens dont il n'est pas toujours possible de discerner la part qui revient au profit et celle qui relève du service. C'est pourtant bien cette relation dialectique, entre d'une part l'immanence économique des attentes du contre-don (en terme de retour sur investissement qu'il s'agisse de profits matériels ou d'image par exemple) et de l'autre les liens de transcendance socio-économiques et culturels (valeurs idéologique, religieuse ou de partage...), qui constitue la dynamique sociale du donner-recevoir dans l'entreprise. À nous de déterminer dans quelle mesure le système du don, qui est à la fois signification (sous le double aspect ambigu de l'offre et du service), notion de valeur et mode particulier de communication, peut se fondre dans la stratégie des entreprises et y engendrer une synergie... 
Les débats de la société en général et de l'entreprise en particulier, sur le bien fondé de l'engagement des entrepreneurs sont le signe apparent d'un profond changement des matrices sociales, culturelles, voire éthiques de l'entreprise. Deux courants contradictoires se côtoient au quotidien et confluent dans la communication des entreprises. D'une part, notre société semble plus que jamais en état de survie, tourmentée par l'individualisme et l'égoïsme, déstabilisée par le chômage et, par ce que les médias ont appelé «la crise ", sorte d'archétype, symbole de toutes les peurs les plus élémentaires de l'homme. D'autre part, de la publicité aux médias en passant par les relais d'opinion, gouvernementaux ou non, tous font appel à la solidarité, mobilisent chacun d'entre nous sous la bannière de l'humanitaire et soudent les discours au nom du respect de la nature ou de l'avenir des hommes... Au cœur de ces antinomies, toute communication d'entreprise qui passe par le registre du don se trouve a fortiori suspecte. L'ambiguïté constitutive du registre du don accompagne l'usage du mot mais caractérise l'acte lui-même et, à partir du moment où les notions de service et d'offre coexistent, rien ne distingue plus une action donatrice de portée philanthropique d'une autre plus mercantile; et pourtant la distinction existe et repose sur le type de communication choisi.

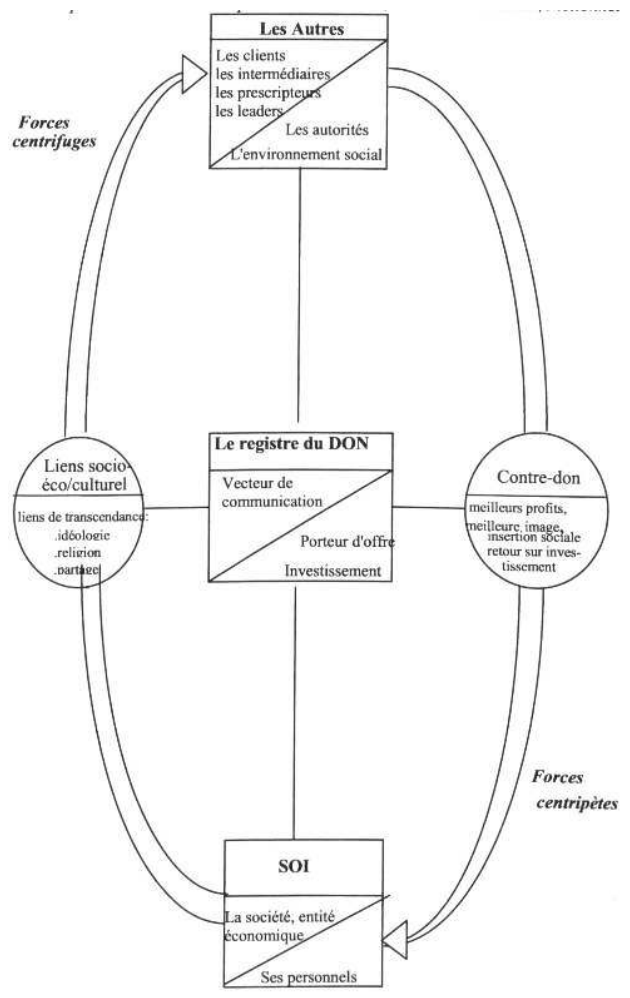

Que l'entreprise joue volontairement ou involontairement de l'ambiguïté inhérente à toute communication s'inscrivant dans le registre du don, importe peu au fond; ce qui est apparu tout à fait déterminant, en revanche, c'est qu'une action donatrice doit être cohérente avec le type de communication choisi. Par effet de capillarité, le caractère oblatif du don et les attributs qui en découlent se transmettent à la marque, au consommateur, à l'entreprise elle-même (personnels et actionnaires); de sorte que, de l'équilibre de la relation dialectique rationalité/charisme nait une dynamique sociale qui constitue la force du don. 
25 Au cœur des intérêts économiques, le mouvement d'empathie mis en circuit contribue à créer des liens de transcendance et à donner sens au système de valeurs partagées à la fois sociales, culturelles et éthiques, de l'entreprise. Les actions donatrices se révèlent un excellent support de la culture d'entreprise, un vecteur d'image efficace et un bon conducteur des objectifs de l'entreprise. Non seulement son engagement n'est ni un phénomène de mode vécu en extériorité, ni un engouement altruiste éphémère, mais il constitue explicitement un élément déterminant de la stratégie de communication d'un autre type de management. d'où l'hypothèse faisant de l'univers $d u$ don, le creuset conceptuel, clé de culture et de civilisation, à la fois signe et symbole, sens et système de son engagement... Même lorsque les entreprises, pour se dégager, parfois non sans difficultés, de l'engrenage trop prégnant du registre du don, créent des fondations, les effets suscités perdurent au sein des politiques de communication. Ces effets se traduisent en terme d'écoute, d'estime et de dialogue mais aussi de participation, d'implication et de responsabilité, sous forme d'une plus grande faculté d'adaptation au changement, par un mouvement d'empathie et de solidarité. D'où les deux courants symétriquement mis en jeu : l'un centrifuge, qui engage une relation avec les autres en tissant des liens aussi bien économiques qu'humanistes; l'autre centripète, qui retourne au donateur, et par une sorte de pouvoir cathartique, l'innocente de ses motivations les plus triviales. On pourra objecter qu'en aucun cas l'entreprise ne résout la question de l'ambiguïté permanente et immanente $d u$ concept $d u$ don. Une telle intention eût été irréaliste. Les pratiques donatrices se fondent dans la stratégie de communication des entreprises avec un sens ambigu (sous le double aspect de l'offre et du service) et une valeur de lien; le registre du don constitue un mode de communication caractérisé, à la fois échange et philosophie d'action; il est l'élément d'un système organisationnel impliquant des relations connotées donnant sens aux produits, aux marchés, aux clients, et combinant d'autres relations d'attente à l'intérieur même de l'entreprise. L'entreprise est contrainte d'évoluer vers cette obligation de partage et de responsabilité. C'est pour elle un question de survie. Dans quelle mesure peut-on vraiment parler de solidarité, de générosité, d'humanisme dans un tel contexte? Bien des fondations d'entreprise ont déjà pris le relais des structures gouvernementales (ou non gouvernementales) afin de pallier, remédier, retarder les conséquences des actuels fléaux économiques, financiers, socioculturels... D’origine américaine, "l'éthique des affaires » a évolué d'un état réactif dans les années soixante à une philosophie d'action dans les années quatre-vingt. Aujourd'hui, s'inscrivant dans le long terme et s'agissant de préserver l'intégrité de la nature et de l'avenir de l'homme, l'éthique des affaires impose " une conduite favorable à tous », une communication transparente, un « principe responsabilité » selon l'expression consacrée par Hans Jonas.

Nous posons, par analogie, que « le principe don » constitue l'étape suivante. Il convient aujourd'hui d'anticiper les événements. La société moderne - notre société de communication - use et abuse de concepts qu'elle vide de leur sens, par médiatisation excessive : que l'on songe aux termes d'identité ou de culture d'entreprise, d'implication ou de participation... Il ne faudrait, à aucun prix, qu'il en advienne ainsi du don. Il ne s'agit pas là d'un concept recette pour managers en quête de performance. Nous n'en sommes encore qu'aux prémices de ce qui devrait devenir une philosophie d'action et une théorie critique de l'économie. Nous songeons tout particulièrement à un travail sur le langage qui tendrait à circonscrire l'étendue des champs lexicaux du don, au regard des clivages sociaux et des variations de ces champs, selon les types de culture en présence. 
L'étude comparée des langages et des pratiques avec d'autres pays devrait apporter aux multinationales, confrontées à l'interpénétration des cultures, des données tangibles pour des actions transnationales. Mais aux côtés du linguiste et de l'ethno-sociologue, c'est à nous, chercheurs en sciences de l'information et de la communication, qu'il convient de mener à bien les observations et les analyses qui permettraient de mettre en évidence le système de communication symbolique sous-tendu par le contractuel. Pour ce faire, de nombreux travaux sont encore nécessaires qui donneront sens et réalité à ce qui a été pour nous une analyse de quelques cas symptomatiques dans une société industrielle en devenir, et ce qui est déjà une sensibilité morale, un mouvement social, une simple aspiration à une vie meilleure.

\section{NOTES}

1. Après une série d'entretiens avec des dirigeants responsables de la communication appartenant à diverses entreprises publiques, privées, associations et agences spécialisées, un questionnaire a été adressé à 329 entreprises avec un retour de 112 réponses exploitables.

\section{RÉSUMÉS}

Les débats sur le bien fondé de l'engagement des entrepreneurs sont le signe d'un profond changement des matrices sociales, culturelles, voire éthiques des entreprises. Cette notion d'engagement constitue un élément déterminant de leur stratégie de communication désignant ainsi un nouveau type de management. D'où l'hypothèse faisant de l'univers $d u$ don, le creuset conceptuel, clé de culture et de civilisation, à la fois signe et symbole, sens et système de cet engagement. Elément d'un système organisationnel, il implique des relations connotées donnant sens aux produits, aux marchés, aux clients... Les pratiques donatrices se fondent dans la stratégie de communication avec un sens ambigu et une valeur de lien, constituant un mode de communication caractérisé, à la fois échange et philosophie d'action.

The debates over the legitimate action of entrepreneurs, are a manifestation of the profound changes occuring in the social, cultural and even ethical domains of the firm. The notion of commitment is a determining factor in corporate communication strategy, and points to a new form of management. The act of patronage is a key to culture and civilisation, both a sign and a symbol, meaning and System. As one of the elements in an organised System, it presupposes connotational relations which impart meaning to products, markets and clients... Corporate patronage is part of the corporate communication strategy, with a double meaning and a link value, constituting a characteristic form of communication, both dialogue and a philosophy of action. 
INDEX

Mots-clés : management, mécénat, langage, don

\section{AUTEUR}

\section{DOMINIQUE KOHLER}

Après des études de lettres comparées à l'université d'État de Milan en Italie et plusieurs années d'enseignement dans cette même université au département Langue et littérature française, Dominique Kohler, actuellement maître de conférences en sciences de l'information et de la communication au CELSA (Paris IV Sorbonne), a poursuivi ses travaux en communication écrite avec une thèse sur les outils de l'écriture et une réflexion appliquée à la communication d'entreprise sur l'écart entre langage et pratique, le dire et le faire. 